\title{
Virtual Endoscopy
}

National Cancer Institute

\section{Source}

National Cancer Institute. Virtual Endoscopy. NCI Thesaurus. Code C20097.

The use of 3D CT or MRI scans to construct a simulated visualization of patient specific organs similar or equivalent to those produced by standard endoscopic procedures.

Virtual endoscopic visualization avoids the risks associated with real endoscopy.

Additionally, there are many body regions not accessible to or compatible with real endoscopy that can be explored with virtual endoscopy. (from R. Robb presentation at Visible Human Project Conference) 